\title{
A GENERALIZED TRANSLATION THEOREM FOR FREE HOMEOMORPHISMS OF SURFACES
}

\author{
LUCIEN GUILLOU
}

(Communicated by Linda Keen)

\begin{abstract}
Let $h$ be a free homeomorphism with a finite set of fixed points on a compact surface. Then $h$ is, on the complement of the fixed point set, everywhere 'semi-locally' conjugate to a translation. This generalizes the Brouwer plane translation theorem.
\end{abstract}

\section{INTRODUCTION}

Let $S$ be a surface, that is, a metric 2-dimensional connected manifold without boundary, and $h$ a homeomorphism of $S$. M. Brown introduced in [B2] the class of free homeomorphisms, that is such that if $h(D) \cap D=\varnothing$ for some 2-disc $D$ in $S$, then $h^{n}(D) \cap D=\varnothing$ for any $n \in \mathbb{Z} \backslash\{0\}$. It is a famous result of Brouwer ([Br], see also [B1], [Fa], [G]) that every homeomorphism of $\mathbb{R}^{2}$ preserving the orientation and without fixed point is free. In fact, Brouwer proved the stronger $([\mathrm{Br}]$, see also $[\mathrm{Fr}],[\mathrm{K}],[\mathrm{G}],[\mathrm{T}])$ :

Plane Translation Theorem. Let $h$ be an orientation preserving fixed point free homeomorphism of $\mathbb{R}^{2}$. Then for every point $p$ in $\mathbb{R}^{2}$ there exists an imbedding $\varphi:\left(\mathbb{R}^{2}, 0\right) \rightarrow\left(\mathbb{R}^{2}, p\right)$ such that

(i) $h \varphi=\varphi \tau$ where $\tau(x, y)=(x+1, y)$,

(ii) on each line $x \times \mathbb{R}, \varphi$ restricts to a proper imbedding into $\mathbb{R}^{2}$, i.e., one with a closed image.

The aim of the present paper is the following generalization of Brouwer's theorem:

Theorem. Let $S$ be a compact surface and $h$ a free homeomorphism of $S$ with a finite set $F$ of fixed points. Then for every point $p$ in $S \backslash F$ there exists an imbedding $\varphi:\left(\mathbb{R}^{2}, 0\right) \rightarrow(S \backslash F, p)$ such that

(i) $h \varphi=\varphi \tau$ where $\tau(x, y)=(x+1, y)$,

(ii) on each line $x \times \mathbb{R}, \varphi$ restricts to a proper imbedding, i.e., $\varphi(x \times \mathbb{R})$ is closed in $S \backslash F$.

Received by the editors March 16, 1994.

1991 Mathematics Subject Classification. Primary 54H20; Secondary 58F99.

Key words and phrases. Brouwer translation theorem, free homeomorphisms. 
When $S$ is the 2-sphere the theorem is due to E. Slaminka [S]. However his proof seems to use strongly Theorem 4.6 of M. Brown [B2], which has no proof (except for fixed point free orientation-preserving homeomorphisms of the plane, cf. [Br, Satz 6] or [G, Lemme 3.8]) and seems to be still an open problem. M. Brown's argument gives only a weaker result (see Proposition 1.5 below); the needed contortions to conclude with this weaker result are explained in $\S 2$ below which elaborates on the proof of Brouwer's theorem given in [G, §4]. (Nevertheless this paper does not depend on [G] and contains, once admitting that orientation preserving fixed point free homeomorphisms of $\mathbb{R}^{2}$ are free, still another (variant of) proof of Brouwer's theorem.) In $\S 3$ we prove the theorem, and in $\S 4$ we extend it to compact surfaces with boundary.

\section{FREE HOMEOMORPHISMS}

We will now recall some basic properties of free homeomorphisms; for more details, see M. Brown [B2].

Let $S$ be a connected surface (in this section $S$ may have a nonempty boundary) and $h$ a free homeomorphism of $S$.

1.1 Definition. A subset $E$ of $S$ such that $h(E) \cap E=\varnothing$ will be called free.

1.2 Proposition. If $E \subset S$ is arc connected and free, then $h^{n}(E) \cap E=\varnothing$ for $n \in \mathbb{Z} \backslash\{0\}$.

Proof. See [B2, Corollary 3.3].

1.3 Definition. A closed simple arc $\alpha$ with endpoints $p$ and $h(p)$ for some $p$ in $S$ is a translation arc if $\alpha \cap h(\alpha)=\{h(p)\}$. The invariant set $L=L(\alpha)=$ $\bigcup_{n \in \mathbf{Z}} h^{n}(\alpha)$ is called a translation line. A translation line has a natural order where $h(\alpha)$ follows $\alpha$.

1.4 Proposition. A translation line $L$ is a submanifold of $S$ homeomorphic to $\mathbb{R}$ (generally not closed).

Proof. That $L$ has no double point follows easily from the freeness of $h$; see Lemma 4.2 of [B2]. To see that $L$ is a submanifold, i.e. that $\bar{L} \backslash L$ is closed, it is enough to show that there exist no translation arc $\beta \subset L$ and no sequence $\left(x_{n}\right)$ with $x_{n} \in L \backslash \beta$ and $x_{n} \rightarrow x \in \stackrel{\circ}{\beta}$. Suppose there is such a sequence and let $x_{n}=h^{p(n)}\left(y_{n}\right)$ with $y_{n} \in \beta$. One can ask that $y_{n} \rightarrow y \in \beta$ for some $y$. Then the subarc $x y$ of $\beta$ is free and we can find a disc $D$ such that $x y \subset \stackrel{\circ}{D}$ and $h(D) \cap D=\varnothing$. But, for $n$ big enough, $x_{n} \in D$ and $y_{n} \in D$, so that $x_{n} \in h^{p(n)}(D) \cap D$ which contradicts the freeness of $h$.

An example where $L$ is not closed was already in Brouwer [Br, Beispiel p. 40]. The same example can also be see in [B2, Figure 7] or [G, Figure 3.4].

1.5 Proposition. Let $L$ be a translation line and $\alpha$ a translation arc generating $L$ (so that $L=L(\alpha)$ ). Let $C$ be an arc connected set such that:

(i) $C$ touches $\alpha$ on at most one side,

(ii) $C$ meets the two components of $L \backslash \alpha$,

(iii) there exists an $n$ such that $C \cap L=C \cap\left(\bigcup_{|i| \leq n} h^{n}(\alpha)\right)$.

Then $C \cap h(C) \neq \varnothing$.

Proof. See the argument in the proof of Theorem 4.6 in [B2]. 
1.6 Remark. Theorem 4.6 of [B2] is the preceding proposition without hypothesis (iii), but in that case $L$ may have accumulation points on $C$ and not in $L$; such a case is overlooked in the given proof of Theorem 4.6. To help clarify a little of this problem, recall the following question of M. Brown:

Let $D$ be a 2-disc such that $h(D) \cap D=\varnothing$ and $f$ an homeomorphism supported in D. Is hf free?

We remark that a positive answer to this question implies the truth of Theorem 4.6 of [B2]. In fact, it is enough to prove the result when $C$ is an arc $\lambda$ not meeting $\alpha$, and, restricting to a subarc of $\lambda$ if necessary, we can suppose that the endpoints ( $p$ and $q$, say, with $q$ following $p$ in the natural order on $L)$ of $\lambda$ cut upon $L$ a subarc $p q$ such that $\lambda \cap p q=\{p, q\}$. Consider now a translation arc $\beta$ for $L$ such that $q \in \stackrel{\circ}{\beta}$ and $\lambda \cap\left(h^{-1}(\beta) \cup \cdots \cup h^{-m+1}(\beta)\right)=\varnothing$ where $m \geq 2$ is such that $p \in h^{-m}(\beta)$. Suppose then by contradiction that $h(\lambda) \cap \lambda=\varnothing$ and let $D$ be a disc neighborhood of $\lambda$ such that $h(D) \cap D=\varnothing$ and $D \cap\left(h^{-1}(\beta) \cup \cdots \cup h^{-m+1}(\beta)\right)=\varnothing$. Let also $f$ be a homeomorphism supported in $D$ such that $f(q)=p$. One has $h(D \cup \beta) \cap \beta=(h(D) \cap \beta) \cup(h(\beta) \cap \beta)=h(\beta) \cap \beta$ so that $h f(\beta) \cap \beta=h(\beta) \cap \beta$ and $\beta$ is a translation arc for $h f$. But $f(q)=p$ and $h^{i}(p) \notin D$ for $1 \leq i \leq m-1$ so that $(h f)^{m}(q)=h^{m}(p) \in \beta$ and $(h f)^{m}(\beta) \cap \beta \neq \varnothing$ which contradicts the freeness of $h f$.

Finally we note that this remark gives another proof of the hypothetical Theorem 4.6 of [B2] for the case of orientation preserving and fixed point free homeomorphisms of the plane. In fact if $h$ is fixed point free, then clearly $h f$ is also fixed point free. Compare to [Br, Satz 6] or [G, Lemme 3.8].

\subsection{Proposition. If $S$ is compact, then $F$ is not empty.}

Proof. See [B2, Lemma 3.4].

1.8 Proposition. There is no annulus $A$ in $S$ with boundary a simple closed curve $\alpha$ and its image $h(\alpha)$ such that $h(A) \cap A=h(\alpha)$.

Proof. We argue by contradiction. If $h^{2}(A)$ does not contain all of $\alpha$ the proof of Lemma 5.2 in [B2] applies and leads to a contradiction with the freeness of $h$. If $h^{2}(A)$ contains $\alpha$, then $S=A \cup h(A) \cup h^{2}(A)$ is a torus or a Klein bottle. Since $h$ is fixed point free on $A$, it is fixed point free on $S$ which is a contradiction to Proposition 1.7.

1.9 Proposition. Any point of $S \backslash F$ is contained in a translation arc if $F$ does not separate $S$.

Proof. See [B2, Lemma 4.1].

\section{AN INDUCTIVE CONSTRUCTION}

Let $p$ be a point of $S \backslash F$ and $\alpha$ a translation arc containing $p$.

2.1 Lemma. There is a triangulation $K$ of $S \backslash F$ such that $\alpha$ is a subcomplex of $K$ and the star of every vertex of $K$ is free.

Proof. We write down $S \backslash F$ as an ascending union of compact bordered surfaces $S_{0} \subset S_{1} \subset S_{2} \subset \cdots$ with $\alpha \subset \stackrel{\circ}{S}_{0}$ and $S_{i} \subset \stackrel{\circ}{S}_{i+1}$. As is well known we can triangulate $S_{0}$ so that $\alpha$ is a subcomplex and the star of every vertex is free. Then we triangulate $S_{1} \backslash \stackrel{\circ}{S}_{0}$ so that the star of every vertex is free and 
the triangulations induced on $\partial S_{0}$ by those of $S_{0}$ and $S_{1} \backslash \stackrel{\circ}{S}_{0}$ are coincident. To satisfy this last condition we may have to subdivide the triangulation of $S_{0}$ near $\partial S_{0}$. We then triangulate $S_{2} \backslash \stackrel{\circ}{S_{1}}$ so that the star of every vertex is free and the triangulations induced on $\partial S_{1}$ by those of $S_{1}$ and $S_{2} \backslash \stackrel{\circ}{S_{1}}$ are coincident. To do this we may have to subdivide the triangulation of $S_{1}$ near $\partial S_{1}$ but can leave it unchanged on $S_{0}$. Therefore, continuing this way, we conclude

2.2 Lemma. Let $K$ be a triangulation of $S \backslash F$ such that the star of every vertex is free and $\alpha$ is a subcomplex of $K$. Then, on each side of $\alpha$, there is a triangle of $K$ adjacent to $\alpha$ which meets neither $h(\alpha)$ nor $h^{-1}(\alpha)$.

Proof. For each triangle $T$ adjacent to $\alpha$, let us consider the triangles included in $T$, of base a side of $T$ in $\alpha \cap T$, and whose third vertex is located on the median of $T$ which bisects (a chosen side of) $\alpha \cap T$. Let $T^{\prime}$ be the largest of those triangles such that $T^{\prime} \cap\left(h(\alpha) \cup h^{-1}(\alpha)\right) \subset \partial T^{\prime}$. We may have to allow $T^{\prime}=\alpha \cap T$ when $T$ contains an end point of $\alpha$. Let us note that if $\stackrel{\circ}{T} \cap\left(h(\alpha) \cup h^{-1}(\alpha)\right)=\varnothing$, then $T^{\prime}=T$. With these notations we have:

\subsection{Assertion. For every such $T^{\prime}, T^{\prime} \cap h^{i}(\alpha)=\varnothing$ for $|i| \geq 2$.}

Proof. Let us suppose that $h^{n}(\alpha),|n| \geq 2$, meets $T^{\prime}$ in a point $x$. We have $x=h^{n}(y)$ for some $y \in \alpha$. The arc made of $\alpha \backslash \alpha \cap T^{\prime}$ and of an arc in $T^{\prime}$ joining the end points of $\alpha \cap T^{\prime}$ without meeting $h^{-1}(\alpha) \cup h(\alpha)$ but going through $x$ (and $y$ if $y \in \alpha \cap T^{\prime}$ ) is a translation arc $\beta$ which contains $x$ and $y$. Therefore $h^{n}(\beta) \cap \beta$ contains $x$ which contradicts Proposition 1.4.

We now show that there exists a $T^{\prime}$ on each side of $\alpha$ such that $T^{\prime} \cap\left(h(\alpha) \cup h^{-1}(\alpha)\right)=\varnothing$.

It follows from the preceding assertion and Proposition 1.5 that any $T^{\prime}$, being free, cannot meet both of $h(\alpha)$ and $h^{-1}(\alpha)$. On the other hand, because of the $T^{\prime}$ containing the end points of $\alpha$, there is on each side of $\alpha$ a $T^{\prime}$ meeting $h(\alpha)$ and another one meeting $h^{-1}(\alpha)$. If all the $T^{\prime}$ on one side of $\alpha$ were meeting $h(\alpha)$ or $h^{-1}(\alpha)$, there would be two consecutive ones, one meeting $h(\alpha)$ and the other $h^{-1}(\alpha)$. But now we see, using the assertion above, that we have a contradiction to Proposition 1.5.

To conclude the proof of Lemma 2.2, it is enough to remark that a $T^{\prime}$ not meeting $h(\alpha)$ or $h^{-1}(\alpha)$ is equal to the $T$ which contains it.

Let us keep with the notation of Lemma 2.2 and let $T_{0}=T$ be a triangle not meeting $h(\alpha) \cup h^{-1}(\alpha)$ given by this lemma. Modifying $\alpha_{0}=\alpha$ by $T_{0}$ we get a new translation arc $\alpha_{1}$ which is still a subcomplex of the triangulation $K$. $\left(\alpha_{1}\right.$ is made of the arc in $\alpha_{0}=p_{0} h\left(p_{0}\right)$ from $p_{0}$ to the first point $r_{0}$ of $T_{0}$, of the arc in $\alpha_{0}$ from the last point $t_{0}$ in $T_{0}$ to $h\left(p_{0}\right)$, and of the arc from $r_{0}$ to $t_{0}$ in $\left.\partial T_{0} \backslash \alpha_{0}\right)$. We can then apply the same lemma again and get a triangle $T_{-1}$ adjacent to $\alpha_{0}$ on the side opposite to $T_{0}$ or a triangle $T_{1}$ adjacent to $\alpha_{1}$ on the side opposite to $T_{0}$. This process can be iterated, and after $n$ applications of Lemma 2.2 we get a sequence $T_{p}, \ldots, T_{0}, \ldots, T_{q}$ of triangles (a priori not necessarily distinct) with $-p+q+1=n, p \leq 0, q \geq 0$, such that $\alpha \cup T_{p} \cup \cdots \cup T_{0} \cup \cdots \cup T_{q}$ is a connected subcomplex of $K$. We also get a sequence $\alpha_{p}, \ldots, \alpha_{0}=\alpha, \ldots, \alpha_{q+1}$ of translation arcs.

According to Assertion 2.3 we have $\left(\alpha \cup T_{0}\right) \cap h\left(\alpha \cup T_{0}\right)=\alpha \cap h(\alpha)$ and $(\alpha \cup$ $\left.T_{0}\right) \cap h^{i}\left(\alpha \cup T_{0}\right)=\varnothing$ for $|i| \geq 2$. I do not know how to show that this situation 
still holds (if it does!) when $T_{0}$ is replaced by $\mathfrak{T}=T_{p} \cup \cdots \cup T_{0} \cup \cdots \cup T_{q}$ without using a hypothetical strong version of Proposition 1.5 (compare to Lemma 6 in $[\mathrm{S}])$. The following lemma nevertheless shows that by exercising more care in the choice of the $T_{i}$ 's we can secure this situation.

2.4 Definition. We shall say that $\alpha \cup \mathfrak{T}$ is critical if $(\alpha \cup \mathfrak{T}) \cap h^{i}(\alpha \cup \mathfrak{T})=\varnothing$ for $|i| \geq 2$ and if $(\alpha \cup \mathfrak{T}) \cap h(\alpha \cup \mathfrak{T})=\alpha \cap h(\alpha)$. Note that this is the case if $n=1$, in which case $\mathfrak{T}=T_{0}$.

2.5 Lemma. Let us suppose that $\alpha \cup \mathfrak{T}$ is critical. Then there exist a triangle $T$ of $K$ adjacent to $\alpha_{q+1}$ on the side opposite to $T_{q}$ and a triangle $U$ of $K$ adjacent to $\alpha_{p}$ on the side opposite to $T_{p}$ such that $\alpha \cup \mathfrak{T} \cup T \cup U$ is still critical. Proof. Let $\widehat{\mathfrak{T}}=\alpha \cup \mathfrak{T}$. As in the proof of Lemma 2.2, to each triangle $T$ adjacent to $\alpha_{q+1}$ on the side opposite to $T_{q}$ we can associate a triangle $T^{\prime} \subset T$ such that $T^{\prime} \cap\left(h^{-1}(\widehat{\mathfrak{T}}) \cup h(\widehat{\mathfrak{T}})\right) \subset \partial T^{\prime}$ and that if $\stackrel{\circ}{T} \cap\left(h^{-1}(\widehat{\mathfrak{T}}) \cup h(\widehat{\mathfrak{T}})\right)=\varnothing$, then $T^{\prime}=T$. One shows as in Assertion 2.3 that such a triangle $T^{\prime}$ satisfies $T^{\prime} \cap h^{i}(\widehat{\mathfrak{T}})=\varnothing$ for $|i| \geq 2$, and one then deduces from Proposition 1.5 that it cannot meet both of $h(\widehat{\mathfrak{T}})$ and $h^{-1}(\widehat{\mathfrak{T}})$. On the other hand, because of the $T^{\prime}$ containing the endpoints of $\alpha_{q+1}$ (which are the same as those of $\alpha$ ), there is a $T^{\prime}$ meeting $h(\widehat{\mathfrak{T}})$ and another one meeting $h^{-1}(\widehat{\mathfrak{T}})$. Using Proposition 1.5 again as in Lemma 2.2 one gets a $T^{\prime}$ not meeting either $h(\widehat{\mathfrak{T}})$ or $h^{-1}(\widehat{\mathfrak{T}})$. Such a $T^{\prime}$ is equal to the $T$ which contains it so that $T \cap h^{i}(\widehat{\mathfrak{T}})=\varnothing$ for all $i \neq 0$ and therefore $\widehat{\mathfrak{T}} \cup T=\alpha \cup \mathfrak{T} \cup T$ is critical. The same reasoning with $\alpha_{p}$ in place of $\alpha_{q+1}$ and $\mathfrak{T} \cup T$ in place of $\mathfrak{T}$ produces the desired $U$.

\section{Proof of THE THEOREM}

We can iterate indefinitely the construction of Lemma 2.5 to get a translation arc $\alpha$ going through $p$ and a doubly infinite sequence $\left\{T_{n}\right\}_{n \in \mathbb{Z}}$ of triangles such that $\alpha \cup\left(\bigcup_{n \in \mathbb{Z}} T_{n}\right)$ is critical. Since each $T_{i}, i \neq-1,0$, meets a $T_{j},|j|<|i|$, or $\alpha$ along an edge and since $\alpha$ consists of a finite number of edges, we can suppose that $T_{i} \cap T_{i+1}$ is always a common edge of $T_{i}$ and $T_{i+1}$ for $i \neq-1$. More precisely we find a subsequence of the $T_{i}$ 's satisfying these properties and reconstruct a sequence of $\alpha_{i}$ exactly as before but using only the $T_{i}$ of the subsequence (and starting from $\alpha=\alpha_{0}$ ). Since $\alpha \cup \mathfrak{T}$ was critical these new $\alpha_{i}$ are certainly translation arcs. In what follows we consider only these new $T_{i}$ 's and $\alpha_{i}$ 's.

3.1 Lemma. The set $\alpha \cup\left(\bigcup_{n \in \mathbb{Z}} T_{n}\right)$ contains no simple closed curve crossing $\alpha$ (which means a curve isotopic, by an arbitrarily small isotopy, to a curve cutting $\alpha$ transversally in one point).

Proof. Let $c$ be such a curve. We first show that it does not bound a disc. If $c=\partial D$ for a 2-disc $D, \alpha$ has one of its end points in $D$ and since $\alpha \cup c$ is critical $h(c)$ (or $h^{-1}(c)$ but we can replace $h$ by $h^{-1}$ if necessary in the following argument) is contained in $\stackrel{\circ}{D}$ and $h^{-1}(c)$ is outside $D$. So that, either $h(D) \subset \stackrel{\circ}{D}$, which contradicts Proposition 1.8 , or $h(D) \cup D$ is a 2-sphere, and $S=S^{2}=D \cup h(D)$ in which case the annulus $A$ bounded by $c$ and $h^{-1}(c)$ has to satisfy $A \cap h(A)=c$ which again contradicts Proposition 1.8. 


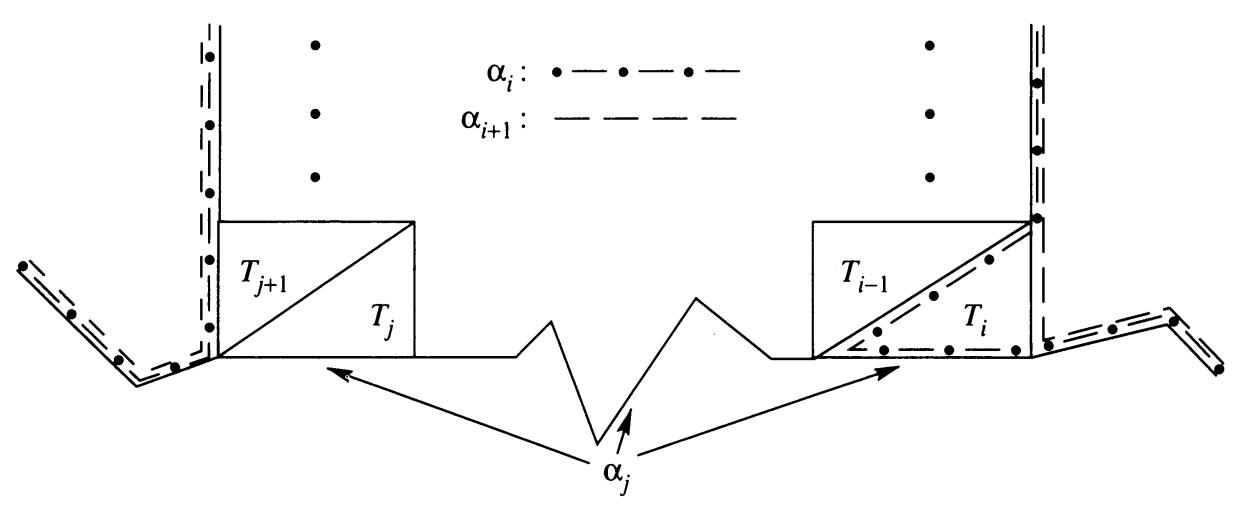

FIGURE 1

Second consider the family $\left\{h^{n}(c)\right\}_{n \in \mathbb{Z}}$ of disjoint simple closed curves. Since none bound a disc there must be a $k>0$ such that $c$ and $h^{k}(c)$ bound an annulus $A$ in $S$. Since $\alpha \cup c$ is critical, if $h(c)$ is contained in $A$ we immediately have a contradiction to Proposition 1.8. If not, then $h(\alpha) \cap A=\varnothing$ so that $h^{-1}(\alpha) \subset A$ and $h^{-1}(c) \subset A$. Once again we arrive at a contradiction to Proposition 1.8.

\subsection{Lemma. The triangles of the family $\left\{T_{n}\right\}_{n \in \mathbb{Z}}$ are all distinct.}

Proof. Let us first show that given a translation arc $\alpha_{j}$ there is no $i \neq j-1$ such that $T_{i}$ and $T_{i+1}$ are on opposite sides of $\alpha_{j}$, that is the sequences $\left\{T_{i}\right\}_{i>j}$ and $\left\{T_{i}\right\}_{i<j}$ may well come back to touch $\alpha_{j}$ but they cannot cross it. We will consider only the case $i>j$ the case $i<j$ being similar. So, suppose there exist such an $\alpha_{j}$ and such a $T_{i}$ and choose them so that $i-j>0$ is minimal. Then $T_{i} \cap T_{k}=\varnothing$ for $j \leq k<i-1$ and all $T_{k}$ 's are distinct for $j \leq k \leq i$. We now look at an imbedded closed band made of $T_{j} \cup T_{j+1} \cup \cdots \cup T_{i}$ and part of a neighborhood of $\alpha_{j}$. It is free and so cannot be a Mœbius band since there are only a finite number of disjoint Mœbius bands in a compact surface. Also $T_{j}$ and $T_{i}$ have to be on the same side of $\alpha_{j}$ for if not we would have a contradiction to Lemma 3.1 which applies to $\alpha_{j}$ since $\alpha_{j} \cup\left(\cup T_{n}\right)_{n \in \mathbb{Z}}$ is critical. Therefore we are left with the situation depicted in Figure 1, which shows that $T_{i} \cap \alpha_{i+1}$ is not contained in $\alpha_{j}$ and so, that $T_{i}$ and $T_{i+1}$ cannot be on opposite sides of $\alpha_{j}$.

As a consequence, all the $T_{i}$ 's, for $i \geq 0$ as well as for $i<0$, are distinct. And we cannot either have $T_{i}=T_{j}$ for some $i \geq 0$ and some $j<0$ since this would lead, using the first part of the proof, to a simple closed curve contradicting Lemma 3.1.

3.3 Lemma. There is a fixed point $q$ (resp. $\left.q^{\prime}\right)$ such that any neighborhood of $q\left(\right.$ resp. $\left.q^{\prime}\right)$ contains all but a finite number of the $T_{i}$ 's for $i>0$ (resp. $\left.i<0\right)$.

Proof. Consider arbitrarily small distinct open balls, one around each fixed point, and call their union $U$. Then $S \backslash U$ is compact and meets only a finite number of $T_{n}$ so there are $k>0$ and $l<0$ so that the connected sets $\bigcup_{n>k} T_{n}$ and $\bigcup_{n<l} T_{n}$ are each contained in a ball.

One can then choose a line $K$ in $\alpha \cup\left(\bigcup_{n \in \mathbb{Z}} T_{n}\right)$ properly embedded in $S \backslash F$ whose ends converge to $q$ and $q^{\prime}$ and crossing $\alpha$. 
3.4 Lemma. The circle $\bar{K} \cup h(\bar{K})$ bounds a 2-disc $D$ in $S$ such that $D \cap h(D)=$ $h(\bar{K})$.

Proof. We cut $S$ along $\bar{K}$ and think of the resulting bordered surface $S^{\prime}$ as a disc with a finite number of bands attached to it. $K$ being free the $\operatorname{arcs} h^{n}(\bar{K})$, $n \neq 0$, have the same endpoints and disjoint interiors, so clearly there are an $s$ and an $r, s \neq r$, such that $h^{s}(\bar{K}) \cup h^{r}(\bar{K})$ bounds a disc in $S^{\prime}$ and therefore an $m$ such that $\bar{K} \cup h^{m}(\bar{K})$ bounds a disc $E$ in $S$. Since $K$ crosses $\alpha$ and since $\alpha \cup K$ is critical, one of the two end points of $\alpha$ is in $E$ and $h(K)$ or $h^{-1}(K)$ is also in $E$. Therefore $\bar{K} \cup h(\bar{K})$ bounds a disc $D$ as required.

The proof of the theorem is now easily completed.

\section{FURTHER REMARKS}

The theorem extends easily to the case of compact surfaces with boundary. Nevertheless we begin with a word of caution: the double of a free homeomorphism may not be free as shown by the following example.

4.1 Example. The homeomorphism of $S^{2}=\mathbb{R}^{2} \cup\{\infty\}$ given by $h(x, y)=$ $(\lambda x, \lambda y)$ for $\lambda \neq 1, \lambda \in \mathbb{R}$, is not free (because of Proposition 1.7) but its restriction to the disc $\mathbb{R}_{+}^{2} \cup\{\infty\}$ is free.

4.2 Theorem. Let $S$ be a compact surface, possibly with boundary, and $h$ a free homeomorphism of $S$ with a finite set $F$ of fixed points. Then for every point $p$ in $S \backslash(\partial S \cup F)$ there exists an imbedding $\varphi:\left(\mathbb{R}^{2}, 0\right) \rightarrow(S \backslash(\partial S \cup F), p)$ such that

(i) $h \varphi=\varphi h$ where $\tau(x, y)=(x+1, y)$,

(ii) on each line $x \times \mathbb{R}, \varphi$ restricts to a proper imbedding, i.e., $\varphi(x \times \mathbb{R})$ is closed in $S \backslash(\partial S \cup F)$.

And for every point $p \in \partial S \backslash F$ there exists an imbedding

$$
\varphi:\left(\mathbb{R}_{+}^{2}, \mathbb{R}_{+}^{2} \backslash \partial \mathbb{R}_{+}^{2}, \partial \mathbb{R}_{+}^{2}, 0\right) \rightarrow(S \backslash F, S \backslash(\partial S \cup F), \partial S \backslash F, p)
$$

such that

(i) $h \varphi=\varphi h$ where $\tau(x, y)=(x+1, y)$,

(ii) on each half-line $x \times[0, \infty)$, $\varphi$ restricts to a proper imbedding, i.e., $\varphi(x \times[0,+\infty))$ is closed in $S \backslash(\partial S \cup F)$

or an imbedding

$$
\varphi:(\mathbb{R} \times[0,1], \mathbb{R} \times(0,1), \mathbb{R} \times\{0,1\}, 0) \rightarrow(S \backslash F, S \backslash(\partial S \cup F), \partial S \backslash F, p)
$$

such that

(i) $h \varphi=\varphi h$ where $\tau(x, y)=(x+1, y)$.

Sketch of proof. We preserve the notation of $\S \S 2$ and 3. If the processes explained in $\$ 2$ can be continued indefinitely, then the proof goes on very similarly to the one given for the boundaryless case. If not, then some $T_{n}$ for (say) $n \leq 0$ has an edge in common with $\partial S$ and the construction can be continued indefinitely for $n>0$, or there is also some $T_{m}, m>0$, which has an edge in common with $\partial S$. In these cases, the line $L$ of $\S 3$ is replaced by a halfline from $\partial S$ to be a fixed point or a closed arc from $\partial S$ to itself. Lemmas similar to Lemma 3.4 then lead to the desired imbeddings. The only new point 
occurs when $p \in S \backslash(\partial S \cup F)$ and $L$ is a half-line from $\partial S$ to a fixed point, in which case we obtain an imbedding $\psi:\left(\mathbb{R}_{+}^{2},(0,1)\right) \rightarrow(S \backslash F, p)$ sending interior into interior and boundary into boundary such that $\psi \tau=\tau \psi$ and that $\psi$ restricted to each half-line $x \times[0,+\infty)$ is proper. We then consider the homeomorphism $\rho: \stackrel{\circ}{\mathbb{R}}_{+}^{2} \rightarrow \stackrel{\circ}{\mathbb{R}}_{+}^{2}$ given by $\rho(x, y)=\left(y+x-1, y^{-1}\right)$; the map $\pi=\psi \rho$ from $\mathbb{R}^{2} \cong \stackrel{\circ}{\mathbb{R}}_{+}^{2}$ to $S \backslash F$ is the imbedding we want.

4.3 Remark. The theorem does not extend as such to noncompact surfaces. In fact, if $S=\mathbb{R}^{2} \backslash \mathbb{Z} \times\{0\}$ and $h(x, y)=(x+1, y)$, then no point of $\mathbb{R} \times\{0\}$ is contained in the image of an imbedding $\varphi$ as in the theorem.

4.4 Remark. The homeomorphism of $S^{2}$ given in Example 4.1 is not free but is, outside of the fixed point set, everywhere 'semi-locally' conjugate to a translation (in the sense of the theorem). This raises the question: Is there a dynamical characterisation of homeomorphisms of compact surfaces (with a finite number of fixed points) everywhere semi-locally conjugate to a translation (outside of the fixed point set)?

\section{ACKNOWLEDGMENT}

I would like to thank P. Greenberg and A. Marin for their careful reading of a preliminary version of this paper.

\section{REFERENCES}

[Br] L. E. J. Brouwer, Beweis des ebenen Translationssatzes, Math. Ann. 72 (1912), 37-54.

[B1] M. Brown, A new proof of Brouwer's lemma on translation arcs, Houston J. Math. 10 (1984), 35-41.

[B2] Homeomorphisms of two dimensional manifolds, Houston J. Math. 11 (1984), 455-469.

[Fa] A. Fathi, An orbit closing proof of Brouwer's lemma on translation arcs, Enseign. Math. 33 (1987), 315-322.

[Fr] J. Franks, A new proof of the Brouwer plane translation theorem, Ergodic Theory Dynamical Systems 12 (1992), 217-226.

[G] L. Guillou, Théorème de translation plane de Brouwer et généralisations du théorème de Poincaré-Birkhoff, Topology 33 (1994), 331-351.

[K] B. de Kerekjarto, The plane translation theorem of Brouwer and the last geometric theorem of Poincare, Acta Sci. Math. Szeged 4 (1928-29), 86-102.

[S] E. E. Slaminka, A Brouwer translation theorem for free homeomorphisms, Trans. Amer. Math. Soc. 306 (1988), 277-291.

[T] H. Terasaka, Ein Beweis des Brouwerschen ebenen Translationssatzes, Japan J. Math. 7 (1930), 61-69.

Universite Grenoble 1, Institut Fourier B.P. 74, Saint-Martin-d'Héres 38402 (Cedex), FRANCE

E-mail address: lguillou@fourier.grenet.fr 